\title{
An Improved Search Space Resizing Method for Model Identification by Standard Genetic Algorithm
}

\author{
*Kumaran Rajarathinam, J. Barry Gomm, DingLi Yu and Ahmed Saad Abdelhadi \\ Mechanical Engineering and Materials Research Centre (MEMARC), Control Systems Group, \\ School of Engineering, Liverpool John Moores University, \\ Byrom Street, Liverpool, L3 3AF, UK \\ *K.Rajarathinam@2011.ljmu.ac.uk
}

\begin{abstract}
In this paper, a new improved search space boundary resizing method for an optimal model's parameter identification by Standard Genetic Algorithms (SGAs) is proposed and demonstrated. The premature convergence to local minima, as a result of search space boundary constraints, is a key consideration in the application of SGAs. The new method improves the convergence to global optima by resizing or extending the upper and lower search boundaries. The resizing of search space boundaries involves two processes, first, an identification of initial value by approximating the dynamic response period and desired settling time. Second, a boundary resizing method derived from the initial search space value. These processes brought the elite groups within feasible boundary regions by consecutive execution and enhanced the SGAs in locating the optimal model's parameters for the identified transfer function. This new method is applied and examined on two processes, a third order transfer function model with and without random disturbance and raw data of excess oxygen. The simulation results assured the new improved search space resizing method's efficiency and flexibility in assisting SGAs to locate optimal transfer function model parameters in their explorations.
\end{abstract}

Keywords—search space boundary resizing; predetermined time constant approximation; genetic algorithms; convergence constraints; premature convergence; transfer function model identification.

\section{INTRODUCTION}

One of the most common problems that may be encountered during model's or control's parameters optimisation by optimisation algorithms is premature convergence due to search space boundary constraints. An optimisation process has prematurely converged to a local optimum if it is no longer able to explore other parts of the search space region than the area currently being explored and there exists another region that may contains a superior solution [1]. Particularly, a set of transfer function parameter's to be optimised for a continuous higher order model distinguishes the dynamic characteristics of the system. At present, numerous well known algorithms and techniques are in application for improving the search space boundary constraints.

Standard Genetic Algorithms (SGAs) are unsophisticated and a very promising approach of an evolutionary computation method in identification of model's parameters. Though, premature convergence is still attributable to the searching space constraints and is a common phenomenon in SGAs [2]. Without prior knowledge of a model's parameters or time constant values, it is highly infeasible to predict the search Upper Space Boundary $\left(\mathrm{SB}_{\text {Upper }}\right)$ and Lower Space Boundary ( $\left.\mathrm{SB}_{\text {Lower }}\right)$. Especially, when the optimum values are located near to the boundary region or outside the boundary region. Insignificant numbers of researches are involved in improvement of searching space to an optimal solution. Based on the complex Box technique, a boundary search method for optimisation problems in the case of the optimal solution at the boundary was proposed [3]. It has been demonstrated and verified, if there is an optimal solution at the boundary constraint set.

Recently, a modified GAs is applied in solving the n-Queens difficulty in chessboard [4]. The holism and random choices cause solving difficulties for SGAs in searching a large space. To improve the solving difficulty, the minimal conflicts algorithm is collaborated with SGAs. The minimal conflicts algorithm gives a partial view for SGAs by a locally searching space. But, the collaboration of algorithms consumed time for searching. A new approach called the self-adaptive boundary search strategy for penalty factor selection within SGAs was proposed [5]. This approach guides the SGA to preserve around constraint boundaries and improves the efficiency of attaining the optimal or near optimal solution. A technique for resolving the structural optimisation difficulties in quantising the subjective uncertainties of active constraints is proposed by fuzzy logic formulation [6]. Another method to improve the prematurity and to sustain the diversity population was proposed by Niche Genetic Algorithm (NGM) associated with isolation mechanism [7]. A comparison study was done on NGM and Annealing Genetic Algorithm where the Annealing Genetic 
Algorithm has better premature convergence [8]. However, the Annealing Genetic Algorithm is time consuming by extra procedures. Another method, named Accelerating Genetic Algorithm (AGM) was proposed to resize the feasible region into the elite individual's adjacent region for better local searching and convergence [9]. Search space boundary reduction for the candidate diameter for each link by pipe index vector and critical path method, along with modified genetic operator's derivatives, was proposed [10] [11]. Further, an improved AGM based on the saddle distribution by which adding random individuals into the initial population to increase the searching ability of optimal solution was proposed [12].

A literature review discloses that most researched techniques are considered based on limited or confined search space boundaries and has an initial knowledge of search space parameters. Also, the discussed research information involves complex mathematical approaches and inevitably can be time consuming for convergence. This paper proposes and investigates a new improved search space method, named the predetermined time constant approximation $\left(T s_{p}\right)$ to enhance the SGAs exploration and exploitation towards the global optima. This method employs a novel search space boundary extension technique by $T s_{p}$ which guides the search to concentrate on optimal values within the boundaries of the feasible region of the solution space. The structure of this paper is as follows; first, the SGAs convergence states for an optimal value by search space boundary constraints are discussed. Second, the approximation process of predetermined time constant methods is discussed. Further, search space boundary extensions for better exploration and for optimal exploitation are discussed here. Finally, the effectiveness of the $T s_{p}$ method is assessed with two processes. Also, the improved AGM based on the saddle distribution method is compared with excess oxygen model to measure the effectiveness of proposed method. The proposed methods are developed and tested in simulations based on Matlab/Simulink models.

\section{POLYNOMIAL COEFFICIENTS}

Consider a system can be modelled by the general order differential equation,

$$
a_{n} \frac{d^{n} y}{d t^{n}}+a_{n-1} \frac{d^{n-1} y}{d t^{n-1}}+\cdots+y=K_{p} f(t-\theta)
$$

where $f(t-\theta)$ is the input signal or forcing function with time delay, $y(t)$ is the output signal and $K_{p}$ is process gain. Assuming zero initial condition, $y(0)=0, y^{\prime}(0)=0$, and taking the laplace transform of equ. 1 gives the general order transfer function is of the form,

$$
G(s)=\frac{Y(s)}{F(s)}=\frac{K_{p}}{a_{n} s^{n}+a_{n-1} s^{n-1}+\cdots+a_{1} s+1} e^{-\theta s}
$$

where $a_{n} \ldots a_{l}$ are coefficients of the denominator polynomial. The denominator polynomial coefficients provide a foundation for determining a system's dynamic response characteristics. In particular the system's poles directly define the components in the homogeneous response. Thus, optimal poles identification is primarily considered here.

\section{CONVERGENCE CONSTRAINTS OF SGAS BY SEARCH SPACE BOUNDARY}

In most situations, selecting the search space boundary regions is delicate if there is no prior knowledge of optimum value location. Thus, a randomly selected search space boundary is a significant factor which leads the SGAs to often converge and get trapped in local optima, resulting in suboptimal solutions. Particularly, it locates near the boundary or outside of the boundary.

As illustrated in Fig. 1, the SGAs convergences by search space boundary constraints can be classified by three states;

- State 1 - If the optimal value $\left(X_{i}\right)$ is located within uniformly distributed elite group around boundary region $\left[X_{i}-\Delta_{G O}, X_{i}+\Delta_{G O}\right]$, the genetic operators have higher probability of converging to global optimum. Thus, the randomly generated initial population within well distributed elite group search boundary has higher probability exploring and exploiting a better parent chromosome. Further, the selected parent chromosome will be evaluated by genetic precision process (selection, crossover and mutation) to produce fitter offspring without any convergence constraint.

- State 2 - If the $X_{i}$ is located near ( $\left[S B_{\text {Lower }}, X_{i}-\Delta_{G O}\right],\left[X_{i}+\right.$ $\left.\left.\Delta_{G O}, S B_{U p p e r}\right]\right)$, the SGAs possibly will converge to local minima. The elite group which is distributed near the boundary may have located a part of the elite group at the outer boundary. If the elite group at the outer part have the genetic information of an optimal value, the genetic operators will suffer to exploit the optimal value and the exploration process will retard. As a result, the search space boundary constraints will lead the SGAs to converge to local minima.

- State 3 - If the $X_{i}$ is located outside the boundary region $\left[S B_{\text {Lower }}>X_{i}>S B_{\text {Upper }}\right]$, the SGAs will fail to explore and exploit the optimal value. The simulation may be retarded and stopped.

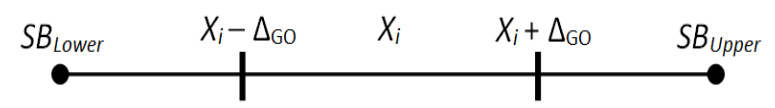

Fig. 1. Schematic diagram of feasible search space boundary region

where $S B_{\text {Lower }}$ is lower search boundary, $S B_{U p p e r}$ is upper search boundary and $\Delta_{G O}$ is the genetic operator for convergence precision.

\section{PREDETERMined TIME CONSTANT APPROXIMATION}

By approximating the distribution of the elite group in a boundary region at the initial stage, gives the genetic operators opportunity to locate the optimal value rapidly without any constraint. To improve searching space boundaries for optimal model identification, a straightforward trial and error technique without a mathematical constraint is introduced here, named 
predetermined time constant approximation $\left(T_{S p}\right)$. The approximation process can be simplified as follows;

- Selecting $\delta T_{s}$, where $\delta$ is the settling band in \%. $(\delta=3,4$ and 5). The selection of desired $\delta$ is according to the raggedness of dynamic response.

- Estimating process's dynamic response period $\left(D R_{P(\tau 2-\tau)}\right)$. At $C(t)=0_{(T=\tau)}$ to $C(t)=1 \pm \delta(\%)_{(T=\tau 2)}$. $(C(t)$ is desired settling point.)

- Approximating an initial $\tau_{l}=D R_{P(\tau 2-\tau)} / \delta$.

- Calculating initial $T_{S p}$ by identified $\tau_{1}$ according to the respective transfer function coefficients $\left(a_{n} s^{n}+a_{n-1} s^{n-1}+\cdots+a_{1} s+1\right)$.

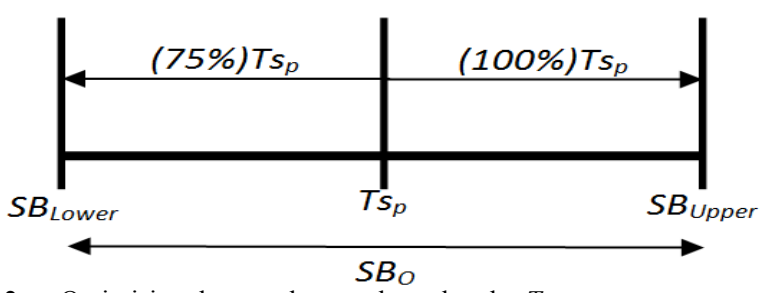

Fig. 2. Optimising the search space boundary by $T_{p}$

$S B_{O}=\left\{S B_{O} ; S B_{\text {Lower }} \leq T_{S_{P}} \leq S B_{\text {Upper }}\right\}$

As illustrated in Fig. 2 and equation 3, the $S B_{O}$ is optimum search space boundary, $S B_{\text {Lower }}$ is lower search boundary and $S B_{U p p e r}$ is upper search boundary. For an $S B_{O}$, the $S B_{U p p e r}$ and $S B_{\text {Lower }}$ are extended by $100 \%$ and $75 \%$ from initial $T_{S p}$, respectively. Especially, $100 \%$ of extension for $S B_{\text {Uper }}$ is required as the optimal solution can be mostly located near to the upper boundary region. Such an extensive search space extension is required for SGAs to explore the elite groups within boundaries and to exploit the $X_{i}$ without any constraint while maintaining the population diversity. Also, such an extension required for characterizing the dynamic homogenous of higher order model parameters.

Generally, the all process of search space boundary adjustment and an optimal $X_{i}$ identification can be stated as follows;

1. Initial attempt - Identified $T_{S p}$ according to the respective transfer function coefficients are applied with $100 \%$ extension on $S B_{\text {Upper }}$. The $S B_{\text {Lower }}$ is extended to approximately $95 \%$ from initial $T_{S p}$ instead of $75 \%$ for better exploration at the beginning stage. Execute the SGAs.

2. Second attempt - Genetically identified $T_{S p}$ of respective transfer function coefficients by initial attempts are extended accordingly ( $S B_{\text {Upper }}$ to $100 \%$ and $S B_{\text {Lower }}$ to $75 \%$ ) to optimise $S B_{O}$. Execute the SGAs.

3. Subsequent attempt - Continuing the SGAs execution with unchanged boundary search approximation by second attempt, until optimal $X_{i}$ and minimum sum of square error (SSE) attained.

4. *Subsequent attempt - If the extended boundary in second attempt is not a $S B_{O}$, consecutive boundary adjustment is essential until $S B_{O}$ is achieved. Then, continuing the SGAs execution until optimal $X_{i}$ and $S S E$ attained.

\section{EXPERIMENTAL RESULTS}

To illustrate the non-complexity and effectiveness, the proposed time constant approximation method is applied on two example processes; a $3^{\text {rd }}$ order transfer function and real data from a process step response.

\section{A. Process $1-3^{\text {rd }}$ Order Transfer Function}

For simulation study, the transfer function of a $3^{\text {rd }}$ order process is selected with process gain $\left(K_{p}=10\right)$,

$$
G(s)=\frac{10}{15 s^{3}+78 s^{2}+6 s+1}
$$

The particular motive of selecting this $3^{\text {rd }}$ order transfer function is that it has a real pole at -5.1245 and a pair of complex poles at $-0.0378 \pm 0.1076 \mathrm{i}$ which are exhibiting a significant oscillatory response. Also, to assess the $T_{S p}$ method's flexibilities and effectiveness, the $3^{\text {rd }}$ order transfer function coefficients are moderately small parameters. So, an appropriate search space boundary extension is required.

According to the $3^{\text {rd }}$ order process step response (Fig. 3), the $D R_{P(\tau 2-\tau)}=123 \mathrm{~s}-0 \mathrm{~s}=123 \mathrm{~s}$. Selecting $\delta T_{s}=5 \mathrm{~T}_{\mathrm{s}}$, as the desired $T_{s}$ is $1 \%$ settling band, gives the initial $\tau_{l}$ is $24.6 \mathrm{~s}$. Therefore, the $T_{S p}$ for the $3^{\text {rd }}$ order polynomial coefficients can be approximated by,

$$
\begin{aligned}
& \tau_{1} s=24.6 ;-\rightarrow\left(T_{1} s\right)^{3}+3\left(T_{1} s\right)^{2}+3 T_{1} s+1 \\
& =14887 s^{3}+1815.5 s^{2}+73.8 s+1
\end{aligned}
$$

According to table 1, the SGAs explored well the entire search space boundaries and exploited the elite group within the chosen boundary region $\left[X_{i}-\Delta_{G O}, X_{i}+\Delta_{G O}\right]$ for $T_{S p}$ values of $S^{2}$ and $S^{l}$ at the initial attempt. This can be seen by the consistency of the $T_{S p}$ values of $S^{2}$ and $S^{l}$ in further execution with readjusted boundaries at the $2^{\text {nd }}$ attempt. This has enhanced the exploitation of an optimal $X_{i}$ at each subsequent attempt by the SGAs.

On other hand, the simulation results reveal that the elite group of $T_{S p}$ values of $S^{3}$ are distributed near to $S B_{\text {Lower }}$ region. This is clearly noticeable at the $1^{\text {st }}, 2^{\text {nd }}$ and $3^{\text {rd }}$ execution results that the $T_{S p}$ value of $S^{3}$ is remaining around $S B_{\text {Lower }}$. This caused the SGAs to fail to exploit an optimal $X_{i}$ and converge to local minima as a part of the elite group is located outside of $S B_{\text {Lower }}$ (state 2). As a result, 3 adjustments on boundaries, especially on $S B_{\text {Lower }}$ are required to optimise the $S B_{O}$ and to bring the elite groups within a feasible boundary region. As expected, the boundaries are optimised and the elite groups are explored well at the $4^{\text {th }}$ execution. Further SGAs execution enhanced an optimal $X_{i}$ exploitation.

The flexibilities and effectiveness of $T_{S p}$ methods is further assessed on $3^{\text {rd }}$ order transfer function model with $5 \%$ disturbance. Initially, identified transfer function coefficients without the disturbance are applied on the $3^{\text {rd }}$ order model with 
disturbance. The simulation result in Fig. 4 and table 2 reveals that the exploration of elite groups and exploitation of an optimal $X_{i}$ for the $3^{\text {rd }}$ order model with disturbance is a very similar process to without disturbance. Thus, the effectiveness of $T_{S p}$ method is well demonstrated in optimizing the $S B_{O}$ and exploiting the $X_{i}$ with or without disturbance. Based on minimum $S S E$, the selected $3^{\text {rd }}$ order model transfer function without disturbance is;

$$
G(s)=\frac{9.997}{21.98 s^{3}+77.68 s^{2}+6.197 s+1}
$$

\begin{tabular}{|c|c|c|c|c|c|c|c|c|c|c|c|}
\hline \multirow{2}{*}{ Execution } & \multicolumn{2}{|c|}{$S^{3}$} & \multicolumn{2}{|c|}{$S^{2}$} & \multicolumn{2}{|c|}{$S^{1}$} & \multirow{2}{*}{$T s_{p}\left(S^{3}\right)$} & \multirow{2}{*}{$T s_{p}\left(S^{2}\right)$} & \multirow{2}{*}{$T s_{p}\left(S^{I}\right)$} & \multirow{2}{*}{$S S E$} & \multirow{2}{*}{ Gen } \\
\hline & $S B_{U}$ & $S B_{L}$ & $\boldsymbol{S B}_{U}$ & $S B_{L}$ & $S B_{U}$ & $S \boldsymbol{B}_{L}$ & & & & & \\
\hline 1 & 29774 & 10 & 3630 & 10 & 148 & 2 & 141.3 & 76.75 & 7.439 & 60.092 & 70 \\
\hline 2 & 280 & 35 & 150 & 20 & 15 & 2 & 42.55 & 77.73 & 6.281 & 8.4924 & 50 \\
\hline 3 & 85 & 12 & 150 & 20 & 15 & 2 & 23.25 & 77.67 & 6.182 & 7.7894 & 30 \\
\hline 4 & 50 & 5 & 150 & 20 & 15 & 2 & 22.98 & 77.69 & 6.179 & 7.7899 & 20 \\
\hline 5 & 50 & 5 & 150 & 20 & 15 & 2 & 21.23 & 77.67 & 6.157 & 7.8149 & 20 \\
\hline 6 & 50 & 5 & 150 & 20 & 15 & 2 & 22.18 & 77.67 & 6.189 & 7.7915 & 30 \\
\hline 7 & 50 & 5 & 150 & 20 & 15 & 2 & 21.98 & 77.68 & 6.197 & 7.6025 & 25 \\
\hline 8 & 50 & 5 & 150 & 20 & 15 & 2 & 21.41 & 77.69 & 6.171 & 7.6171 & 35 \\
\hline 9 & 50 & 5 & 150 & 20 & 15 & 2 & 23.53 & 77.67 & 6.186 & 7.7898 & 25 \\
\hline 10 & 50 & 5 & 150 & 20 & 15 & 2 & 22.62 & 77.68 & 6.175 & 7.7914 & 15 \\
\hline 11 & 50 & 5 & 150 & 20 & 15 & 2 & 23.49 & 77.69 & 6.183 & 7.7895 & 20 \\
\hline
\end{tabular}

TABLE II. SimUlation RESUlts OF $3^{\text {RD }}$ ORDER TRANSFER FUnCTION With 5\% DistURBANCE EXECUTIONS

\begin{tabular}{|c|c|c|c|c|c|c|c|c|c|c|c|}
\hline \multirow{2}{*}{ Execution } & \multicolumn{2}{|c|}{$S^{3}$} & \multicolumn{2}{|c|}{$S^{2}$} & \multicolumn{2}{|c|}{$S^{1}$} & \multirow{2}{*}{$T s_{p}\left(S^{3}\right)$} & \multirow{2}{*}{$T \boldsymbol{s}_{p}\left(S^{2}\right)$} & \multirow{2}{*}{$T \boldsymbol{s}_{p}\left(\boldsymbol{S}^{l}\right)$} & \multirow{2}{*}{$S S E$} & \multirow{2}{*}{ Gen } \\
\hline & $S B_{U}$ & $S B_{L}$ & $S B_{U}$ & $S B_{L}$ & $S B_{U}$ & $S B_{L}$ & & & & & \\
\hline 1 & 29774 & 10 & 3630 & 10 & 148 & 2 & 380.4 & 82.03 & 11.27 & 150.832 & 90 \\
\hline 2 & 760 & 95 & 165 & 20 & 22 & 3 & 95.15 & 77.78 & 6.296 & 60.1486 & 78 \\
\hline 3 & 190 & 24 & 155 & 20 & 13 & 2 & 25.29 & 77.57 & 6.211 & 33.4558 & 43 \\
\hline 4 & 50 & 6 & 155 & 20 & 13 & 2 & 24.02 & 77.57 & 6.196 & 33.4456 & 37 \\
\hline 5 & 50 & 6 & 155 & 20 & 13 & 2 & 24.67 & 77.58 & 6.049 & 33.4481 & 32 \\
\hline 6 & 50 & 6 & 155 & 20 & 13 & 2 & 24.05 & 76.33 & 6.398 & 33.4452 & 28 \\
\hline 7 & 50 & 6 & 155 & 20 & 13 & 2 & 26.14 & 77.91 & 6.215 & 33.4627 & 22 \\
\hline 8 & 50 & 6 & 155 & 20 & 13 & 2 & 24.25 & 77.51 & 6.198 & 33.4459 & 30 \\
\hline 9 & 50 & 6 & 155 & 20 & 13 & 2 & 22.99 & 77.58 & 6.186 & 33.4503 & 21 \\
\hline 10 & 50 & 6 & 155 & 20 & 13 & 2 & 22.89 & 77.58 & 6.183 & 33.4511 & 42 \\
\hline 11 & 50 & 6 & 155 & 20 & 13 & 2 & 22.76 & 77.84 & 6.114 & 33.4596 & 34 \\
\hline
\end{tabular}

TABLE III. SIMULATION RESUlTS OF EO $\mathrm{E}_{2}$ EXECUTIONS

\begin{tabular}{|c|c|c|c|c|c|c|c|c|c|c|c|}
\hline \multirow{2}{*}{ Execution } & \multicolumn{2}{|c|}{$S^{3}$} & \multicolumn{2}{|c|}{$S^{2}$} & \multicolumn{2}{|c|}{$S^{I}$} & \multirow{2}{*}{$T s_{p}\left(S^{3}\right)$} & \multirow{2}{*}{$T s_{p}\left(S^{2}\right)$} & \multirow{2}{*}{$T s_{p}\left(S^{I}\right)$} & \multirow{2}{*}{$S S E$} & \multirow{2}{*}{ Gen } \\
\hline & $S B_{U}$ & $S B_{L}$ & $S B_{U}$ & $S B_{L}$ & $S B_{U}$ & $S B_{L}$ & & & & & \\
\hline 1 & $3.5 \mathrm{e} 6$ & 10 & $8.6 \mathrm{e} 4$ & 10 & $7.2 \mathrm{e} 2$ & 10 & 8088.2 & 10085 & 178.73 & 0.86796 & 70 \\
\hline 2 & $1.6 \mathrm{e} 4$ & $2 \mathrm{e} 3$ & $2 \mathrm{e} 4$ & $2 \mathrm{e} 3$ & $3.5 \mathrm{e} 2$ & 40 & 4039.7 & 14074 & 180.02 & 0.49128 & 20 \\
\hline 3 & $1.6 \mathrm{e} 4$ & $2 \mathrm{e} 3$ & $2 \mathrm{e} 4$ & $2 \mathrm{e} 3$ & $3.5 \mathrm{e} 2$ & 40 & 2699.7 & 13304 & 180.38 & 0.51873 & 40 \\
\hline 4 & $1.6 \mathrm{e} 4$ & $2 \mathrm{e} 3$ & $2 \mathrm{e} 4$ & $2 \mathrm{e} 3$ & $3.5 \mathrm{e} 2$ & 40 & 4875.7 & 14995 & 183.64 & 0.49413 & 40 \\
\hline 5 & $1.6 \mathrm{e} 4$ & $2 \mathrm{e} 3$ & $2 \mathrm{e} 4$ & $2 \mathrm{e} 3$ & $3.5 \mathrm{e} 2$ & 40 & 8187.7 & 14524 & 181.41 & 0.48654 & 20 \\
\hline 6 & $1.6 \mathrm{e} 4$ & $2 \mathrm{e} 3$ & $2 \mathrm{e} 4$ & $2 \mathrm{e} 3$ & $3.5 \mathrm{e} 2$ & 40 & 8079.1 & 16513 & 184.16 & 0.53421 & 35 \\
\hline 7 & $1.6 \mathrm{e} 4$ & $2 \mathrm{e} 3$ & $2 \mathrm{e} 4$ & $2 \mathrm{e} 3$ & $3.5 \mathrm{e} 2$ & 40 & 4330.5 & 14555 & 177.2 & 0.5109 & 90 \\
\hline 8 & $1.6 \mathrm{e} 4$ & $2 \mathrm{e} 3$ & $2 \mathrm{e} 4$ & $2 \mathrm{e} 3$ & $3.5 \mathrm{e} 2$ & 40 & 4137.2 & 15028 & 181.88 & 0.48758 & 22 \\
\hline 9 & $1.6 \mathrm{e} 4$ & $2 \mathrm{e} 3$ & $2 \mathrm{e} 4$ & $2 \mathrm{e} 3$ & $3.5 \mathrm{e} 2$ & 40 & 9903.9 & 16043 & 182.3 & 0.51771 & 80 \\
\hline
\end{tabular}




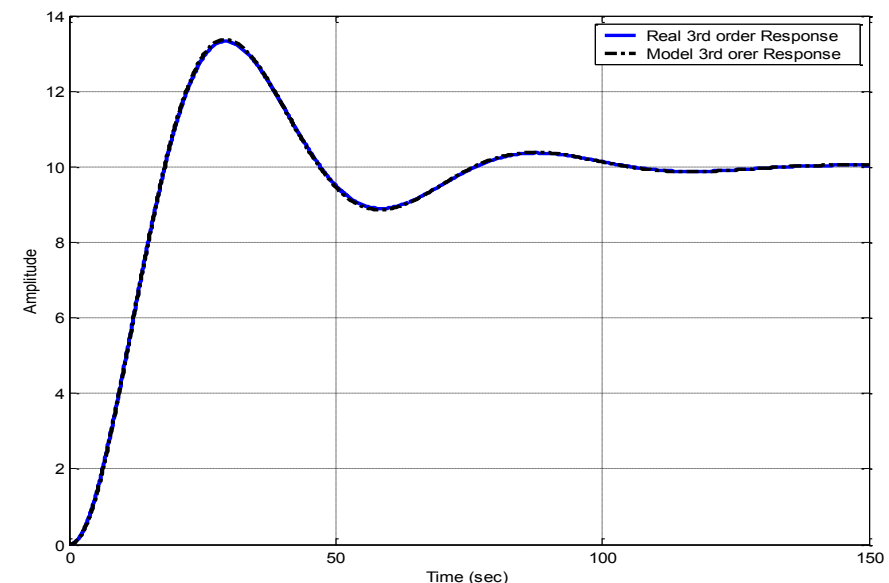

Fig. 3. Transient response of $3^{\text {rd }}$ order transfer function real and model process

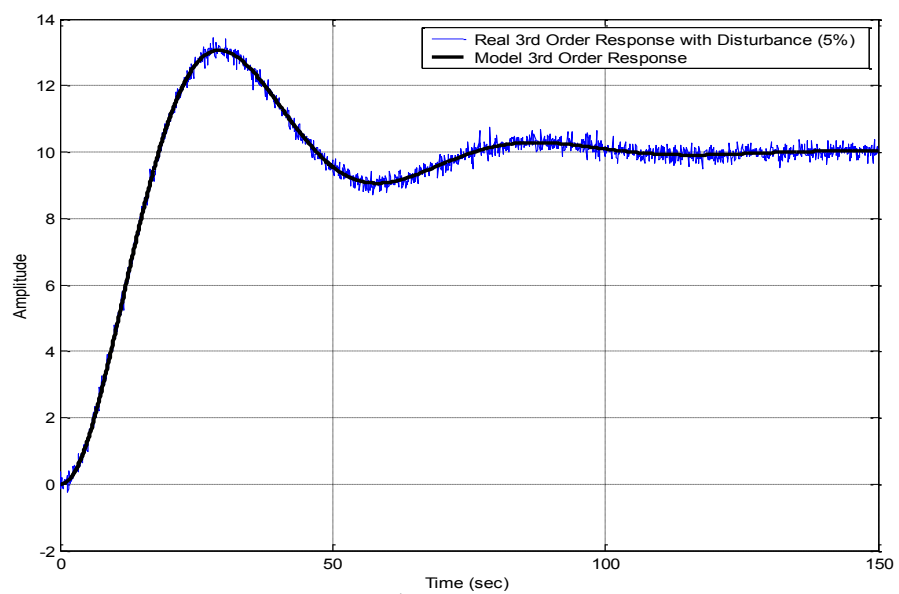

Fig. 4. Transient responses of $3^{\text {rd }}$ order transfer function real and model with $5 \%$ disturbance

and with $5 \%$ disturbance is;

$$
G(s)=\frac{9.976}{24.05 s^{3}+76.33 s^{2}+6.398 s+1}
$$

By comparing the identified $T_{S p}$ coefficients with $3^{\text {rd }}$ order transfer function model's parameters, the $S^{2}$ and $S^{l}$ values have $98 \%$ similarity. But, the $S^{3}$ value only has $54 \%$ of similarity. According to table 4 and Fig. 5, the complex poles of all $3^{\text {rd }}$ order models illustrate that the imaginary parts are considerably constant. But, the real part is slightly moved along the real axis causing a small change in the damping ratio for these roots. These small changes in the complex poles are consolidated with the differing position of the other real root.

\begin{tabular}{|c|c|c|c|} 
TABLE IV. & \multicolumn{3}{c|}{ ROOTS OF $3^{\mathrm{RD}}$ ORDER MODEL'S } \\
\begin{tabular}{|c|c|c|c|}
\hline Model's & $\boldsymbol{S}^{3}$ & $\boldsymbol{S}^{\mathbf{2}} \boldsymbol{\&} \boldsymbol{S}^{\boldsymbol{1}}$ & Damping Ratio \\
\hline Real & -5.1245 & $-0.0378 \pm 0.1076 \mathrm{i}$ & 0.331 \\
\hline Without Disturbance & -3.4564 & $-0.0389 \pm 0.1079 \mathrm{i}$ & 0.339 \\
\hline With Disturbance & -3.0921 & $-0.0408 \pm 0.1085 \mathrm{i}$ & 0.352 \\
\hline
\end{tabular}
\end{tabular}

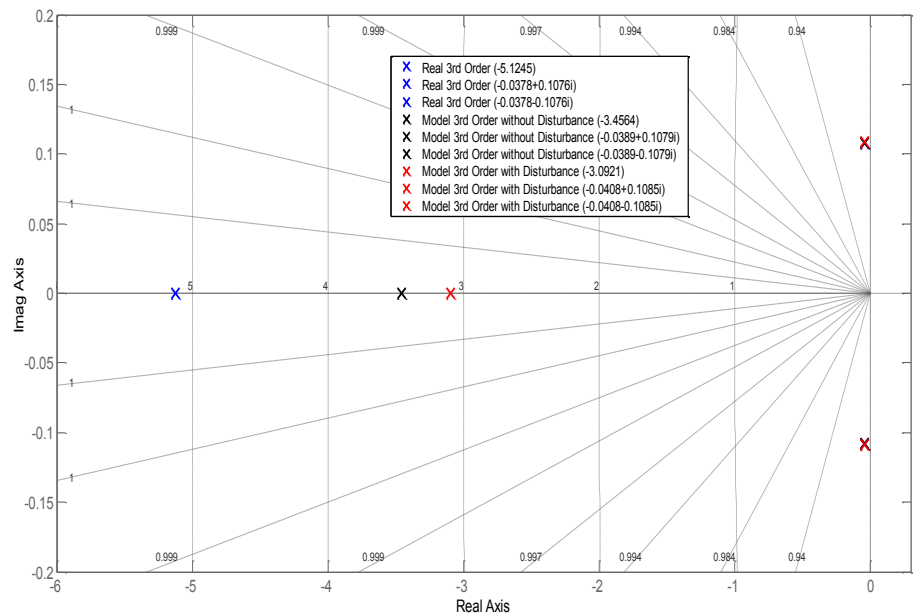

Fig. 5. Roots of $3^{\text {rd }}$ order transfer function real and model with $5 \%$ disturbance

Notice that the peak time is the same for all waveforms because the imaginary part remains the same. Nevertheless, the identified model responses, with and without noise, closely match the response of the actual system as shown in Figs. 3 and 4.

\section{B. Process 2 - Excess Oxygen $\left(\mathrm{EO}_{2}\right)$}

A raw numerical data of excess oxygen $\left(\mathrm{EO}_{2}\right)$ is collected from a real industrial furnace by empirical technique for 1000 seconds with 5 seconds interval. As illustrated in Fig. 6, the process response of $\mathrm{EO}_{2}$ is exhibiting an approximate first-order plus dead-time (FOPDT) dynamic system. The data was gathered by the step input of increasing air ratio from 9.5 to 10.5 in volumetric.

As discussed earlier, the time constant $\left(\tau_{S}\right)$ of transfer function are primarily considered here for optimal model identification by $T_{S p}$ method. Whereas, the process gain $\left(K_{p}\right)$ and transport delay $(\theta)$ can be approximated by close observation of the transient response. As illustrated on the transient response of $\mathrm{EO}_{2}, K_{p} \approx 1.54$ and $\theta \approx 160$ s. As a result, an extension on the search space boundaries are approximated for $K_{p} \in[1: 2]$ and $\theta \in[50: 200]$.

According to the $\mathrm{EO}_{2}$ response, the $D R_{P(\tau 2-\tau)}=700 \mathrm{~s}-100 \mathrm{~s}=$ $600 \mathrm{~s}$. Selecting $\delta T_{s}=5 \mathrm{~T}_{\mathrm{s}}$, as the desired $T_{s}$ is $1 \%$ settling band, gives the initial $\tau_{1}$ as $120 \mathrm{~s}$. For $\mathrm{EO}_{2}$, the selection of an optimal

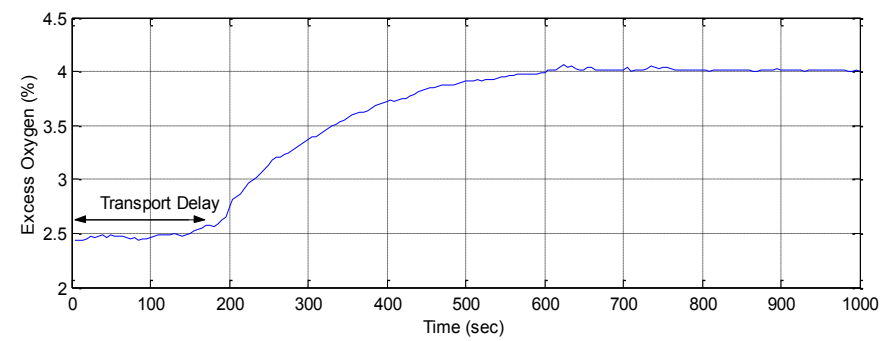

Fig. 6. Step response of $\mathrm{EO}_{2}$ 


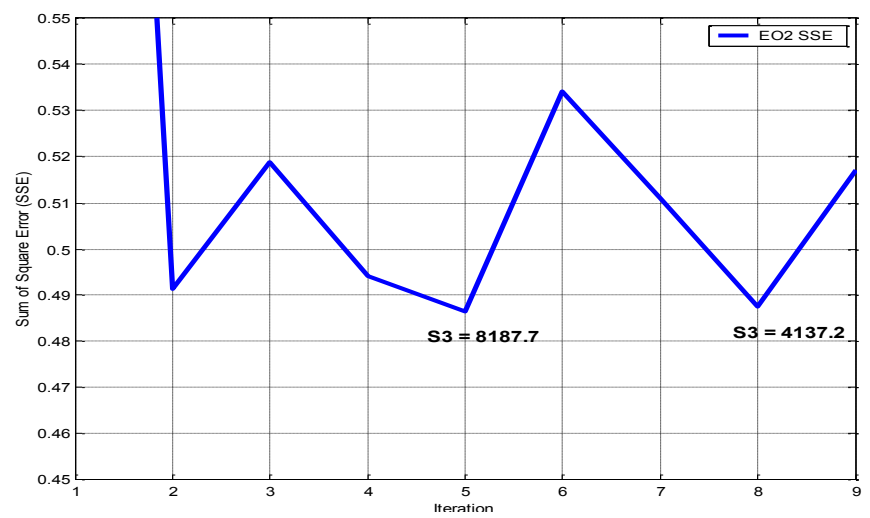

Fig. 7. Two optimal values of $S^{3}$ for $\mathrm{EO}_{2}$

model is a $3^{\text {rd }}$ order transfer function. Therefore, the $T_{S p}$ for the $3^{\text {rd }}$ order polynomial coefficients can be approximated,

$$
\begin{aligned}
& \tau_{1} s=120 ;-\rightarrow\left(T_{1} s\right)^{3}+3\left(T_{1} s\right)^{2}+3 T_{1} s+1 \\
& =1.728 e^{6} s^{3}+4.32 e^{4} s^{2}+3.6 e^{2} s+1
\end{aligned}
$$

As illustrated in table 3, the distribution of elite groups within boundary region $\left[X_{i}-\Delta_{G O}, X_{i}+\Delta_{G O}\right]$, the exploitation of optimal $X_{i}$ and the consistency of the $T_{S p}$ values of $S^{2}$ and $S^{l}$ in further execution by SGAs are exhibiting similar process characteristics as $3^{\text {rd }}$ transfer function model.

Based on the initial attempt, the elite groups of $T_{S p}$ value of $S^{3}$ are uniformly distributed around $X_{i}-\Delta_{G O}$ region. As illustrated in table 3 , the $T_{S p}$ value of $S^{3}$ is still continuously evolving within the boundary $S B_{O}$ region at each execution. Therefore, further readjustment of $S B_{O}$ boundaries is not required as the elite groups are still within the boundary range (state 1) as discussed in section 3. For this $3^{\text {rd }}$ order model of $\mathrm{EO}_{2}$, the $T_{S p}$ values by the $5^{\text {th }}$ execution are selected as the $S S E$ and Gen (generation) is minimum and optimal. The identified transfer function is,

$$
G(s)_{E O_{2}}=\frac{1.555}{8187.7 s^{3}+14524 s^{2}+181.41 s+1} e^{-109.36 s}
$$

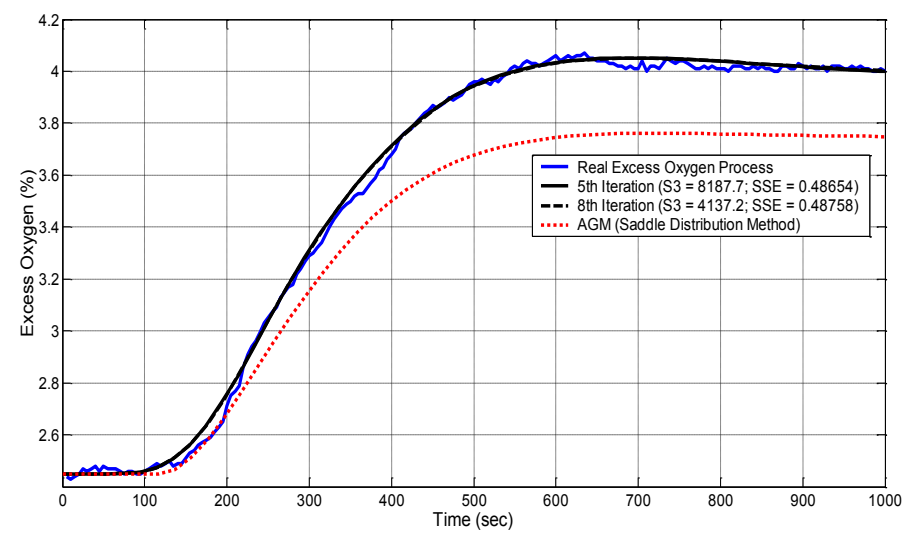

Fig. 8. Transient responses of 2 global optimal values with real process of $\mathrm{EO}_{2}$
However, the inconsistency of $S^{3}$ shows that there are two optimal values of $X_{i}\left(X_{i}=8187.7 ; 4137.2\right)$, which frequently appear within the $S B_{O}$ region at $1^{\text {st }}, 2^{\text {nd }}, 4^{\text {th }}, 5^{\text {th }}, 6^{\text {th }}, 7^{\text {th }}$ and $8^{\text {th }}$ execution. This has been verified by simulation results in Fig. 7 and 8 of both optimal $X_{i}$ values of $S^{3}$ and minimum SSE. Further, the improved AGM based on saddle distribution method is suffered to optimise the search space region and to characterise the homogeneous response of higher order polynomial coefficients as the AGM method is usually suitable for confined search space region.

\section{CONCLUSION}

The proposed predetermined time constant $\left(T_{S p}\right)$ method enhanced the optimization of search space boundaries for global optima convergence. The response's dynamic period and settling time provide better presumption of an initial $T_{S p}$ for search space optimisation. The extended $S B_{\text {Upper }}$ and $S B_{\text {Lower }}$ for an optimal search boundary $\left(S B_{O}\right)$ derived from an initial $T_{S p}$ brought the elite group within a feasible bounded search region. Further, SGAs execution improved the exploration of elite groups to locate and exploit the optimal values for the identified model parameters. As expected, the polynomial coefficients (for $S^{l}, S^{2}$ and $S^{3}$ ) of two processes are optimised well by SGAs.

\section{References}

[1] R.K. Ursem, "Models for Evolutionary Algorithms and Their Applications in System Identification and Control Optimisation", $\mathrm{PhD}$ Thesis, University of Aarhus, Denmark, 2003.

[2] J.H. Holland, "Adaptation in Nature and Artificial System", Ann Arbor: The University of Michigan Press, 1975.

[3] B.F. Zhu, Z.M. Li and B.C. Zhang, "Structural Optimal Design: Theory and Applications", Hydro-Electrical Press, Beijing, China, 1984.

[4] J.E.A. Heris and M.A. Oskoei, "Modefied Genetic Algorithm for solving N-Queens Problem", Iranian Conf. Intel. Sys. (ICIS), pp. 1-5, 2014.

[5] Z.Y. Wu and A.R. Simpson, "A Self-Adaptive Boundary Search Genetic Algorithm and its Application to Water Distribution Systems", Journal of Hydraulic Research, Vol. 40, Issue (2), pp. 191-203, 2002.

[6] Z.Y. Wu and Y.T. Wang, "Arch Dam Optimisation Design Under Strength Fuzziness and Fuzzy Safety Measure", Proc. Of Int. Conf. on Arch Dam, Hehai University, Nanjing, China, pp. 129-131, 1992.

[7] Y. Lin, J.M. Hao, Z.S. Ji and Y.S. Dai, "A Study of Genetic Algorithm based on Isolation Niche Technique", Journal of Systems Eng., Vol. 15, pp. 86-91, 2000.

[8] Q.Y. Tu and Y.D. Mei, "Comparison of Genetic Simulated Annealing Algorithm and Niche Genetic Algorithm for reservoir Optimal Operation", Hydropower Automation and Dam Monitoring, Vol. 32, pp. 1-4, 2008.

[9] J.L. Jin, X.H. Yang and J. Ding, "An Improved Simple Genetic AlgorithmAccelerating Genetic Algorithm", Systems Eng. Theory \& Practice, pp. 813, 2001.

[10] K.S. Mahendra, R. Gupta and P.R. Bhave, "Optimal Design of Water Networks using Genetic Algorithm with Reduction in Search Space", Journal Water Resour. Plann. Mngmnt., ASCE, Vol. 134, Issue (2), pp. 147-160, 2008

[11] K. Vairavamoorthy and M. Ali, "Pipe Index Vector: A Method to Improve Genetic Algorithm-Based Pipe Optimisation", Journal Hydraulic Engg, ASCE, Vol. 131, Issue (12), pp. 1117-1125, 2005.

[12] B. Xu, P. Zhong and L. Tang, "Improvement on Boundary Searching of Accelerating Genetic Algorithm", Inter. Conf. on Intelligent Design and Engineering Application”, pp.301-305, 2012. 
